\title{
Analytical-numerical method for attractor localization of generalized Chua's system
}

\author{
Kuznetsov N.V., Leonov G.A., Vagaitsev V.I. \\ Draft $^{1} 2$
}

\begin{abstract}
Methods of harmonic linearization \& describing function, numerical methods, and the applied bifurcation theory together discover new opportunities for analysis of periodic oscillations of control systems. In the present paper these opportunities are demonstrated. New analytical-numerical method based on the above-mentioned technique is discussed. Application of this technique for hidden attractor (basin of attraction of which does not contain neighborhoods of equilibria) localization of generalized Chua's systems is given.
\end{abstract}

Keywords: chaotic hidden attractor, smooth Chua system, Chua circuits, hidden oscillation, describing function method

\section{Introduction}

In the works an effective methods of search of periodic solutions of multidimensional nonlinear dynamical systems with scalar nonlinearity were suggested. In the present work the approach suggested is generalized on the systems of the form

$$
\frac{d \mathbf{x}}{d t}=\mathbf{P} \mathbf{x}+\boldsymbol{\psi}(\mathbf{x})
$$

where $\mathbf{P}$ is a constant $n \times n$-matrix, $\boldsymbol{\psi}(\mathbf{x})$ is a continuous vector-function, and $\boldsymbol{\psi}(0)=0$. It is shown that modification of the used algorithms permits one to fulfil localization of attractors of system (1), which were obtained by Chua and his progeny in studying nonlinear electrical circuits with feedback [|Chua \& Lin(1990), Chua(1992a), Chua(1992b), Bilotta \& Pantano(2008), Chen \& Ueta(2002)|].

For the search of periodic solution close to harmonic oscillation, we consider matrix $\mathbf{K}$ such that the matrix $\mathbf{P}_{0}=\mathbf{P}+\mathbf{K}$ has a pair of purely imaginary eigenvalues $\pm i \omega_{0}\left(\omega_{0}>0\right)$ and the rest of its eigenvalues have negative real parts. Then system (1) can be rewritten as

$$
\frac{d \mathbf{x}}{d t}=\mathbf{P}_{0} \mathbf{x}+\varphi(\mathbf{x})
$$

where $\boldsymbol{\varphi}(\mathbf{x})=\boldsymbol{\psi}(\mathbf{x})-\mathbf{K} \mathbf{x}$.

Since we are interested in periodic solutions of system (2), it is natural to introduce a finite sequence of continuous functions $\varphi^{0}(\mathbf{x}), \varphi^{1}(\mathbf{x}), \ldots, \varphi^{m}(\mathbf{x})$ in such a way that the graphs of neighboring functions $\varphi^{j}$ and $\varphi^{j+1}$, in a sense, are slightly differed from each other, the function $\varphi^{0}(\mathbf{x})$ is small, and $\varphi^{m}(\mathbf{x})=\varphi(\mathbf{x})$.

In this case the smallness of function $\varphi^{0}(\mathbf{x})$ permits one to apply and justify the method of harmonic linearization for the system

$$
\frac{d \mathbf{x}}{d t}=\mathbf{P}_{0} \mathbf{x}+\varphi^{0}(\mathbf{x})
$$

\footnotetext{
${ }^{1}$ Nikolay V. Kuznetsov, nkuznetsov239 at gmail.com (correspondence author)

${ }^{2} \mathrm{PDF}$ slides http://www.math.spbu.ru/user/nk/PDF/Hidden-attractor-localization-Chua-circuit.pdf
} 
if the stable periodic solution $\mathbf{x}^{0}(t)$ close to harmonic one is determined. All the points of this stable periodic solution are located in the domain of attraction of stable periodic solution $\mathbf{x}^{1}(t)$ of the system

$$
\frac{d \mathbf{x}}{d t}=\mathbf{P}_{0} \mathbf{x}+\varphi^{j}(\mathbf{x})
$$

with $j=1$ or when pass from (3) to system (4) with $j=1$, we observe the instability bifurcation destroying periodic solution. In the first case it is possible to find $\mathbf{x}^{1}(t)$ numerically, starting a trajectory of system (4) with $j=1$ from the initial point $\mathbf{x}^{0}(0)$.

Starting from the point $\mathbf{x}^{0}(0)$, after transient process the computational procedure reaches to the periodic solution $\mathbf{x}^{1}(t)$ and computes it. In this case the interval $(0, \widetilde{T})$, on which the computation is carried out, must be sufficiently large.

After the computation of $\mathbf{x}^{1}(t)$ it is possible to obtain the following system (4) with $j=2$ and to organize a similar procedure of computing the periodic solution $\mathbf{x}^{2}(t)$, starting a trajectory, which with increasing $t$ approaches to periodic trajectory $\mathbf{x}^{2}(t)$, from the initial point $\mathbf{x}^{2}(0)=\mathbf{x}^{1}(\widetilde{T})$.

Proceeding this procedure and computing $\mathbf{x}^{j}(t)$, using trajectories of system (4) with the initial data $\mathbf{x}^{j}(0)=\mathbf{x}^{j-1}(\widetilde{T})$, we either arrive at periodic solution of system (4) with $j=m$ (i.e., at original system (2)) either observe, at a certain step, the instability bifurcation destroying periodic solution.

In the described procedure the simplest and the most natural class of functions $\varphi^{j}$ are the following functions: $\boldsymbol{\varphi}^{0}(\mathbf{x})=\varepsilon \boldsymbol{\varphi}(\mathbf{x}), \boldsymbol{\varphi}^{1}(\mathbf{x})=\varepsilon^{1} \boldsymbol{\varphi}(\mathbf{x}), \ldots, \boldsymbol{\varphi}^{m-1}(\mathbf{x})=\varepsilon^{m-1} \boldsymbol{\varphi}(\mathbf{x}), \boldsymbol{\varphi}^{m}(\mathbf{x})=\boldsymbol{\varphi}(\mathbf{x})$, where $\varepsilon$ is a "classical" small positive parameter and, for example, $\varepsilon^{j}=j / m, j=1, \ldots, m$.

For system (3) with such function $\varphi^{0}(\mathbf{x})$ it turns out that it is possible to justify rigorously the method of harmonic linearization and to determine the initial conditions, for which system (3) has a stable periodic solution close to harmonic one.

\section{Poincare map for harmonic linearization}

By nonsingular linear transformation system (3) can be reduced to the form

$$
\begin{aligned}
& \dot{x}_{1}=-\omega_{0} x_{2}+\varepsilon \varphi_{1}\left(x_{1}, x_{2}, \mathbf{x}_{3}\right), \\
& \dot{x}_{2}=\omega_{0} x_{1}+\varepsilon \varphi_{2}\left(x_{1}, x_{2}, \mathbf{x}_{3}\right), \\
& \dot{\mathbf{x}}_{3}=\mathbf{A x}_{3}+\varepsilon \boldsymbol{\varphi}_{3}\left(x_{1}, x_{2}, \mathbf{x}_{3}\right)
\end{aligned}
$$

Here $\mathbf{A}$ is a constant $(n-2) \times(n-2)$ matrix, all eigenvalues of which have negative real parts, $\boldsymbol{\varphi}_{3}$ is an $(n-2)$-dimensional vector-function, $\varphi_{1}, \varphi_{2}$ are certain scalar functions. Without loss of generality, it may be assumed that for the matrix $\mathbf{A}$ there exists positive number $\alpha>0$ such that

$$
\mathbf{x}_{3}^{*}\left(\mathbf{A}+\mathbf{A}^{*}\right) \mathbf{x}_{3} \leq-2 \alpha\left|\mathbf{x}_{3}\right|^{2}, \quad \forall \mathbf{x}_{3} \in \mathbb{R}^{n-2}
$$

Here $*$ is a transposition operation.

In a phase space of system (5) we introduce the following set

$$
\Omega=\left\{\left|\mathbf{x}_{3}\right| \leq D \varepsilon, \quad x_{2}=0, \quad x_{1} \in\left[a_{1}, a_{2}\right]\right\} .
$$

Here $D, a_{1}, a_{2}$ are certain positive numbers, which will be determined below.

Define $n$-dimensional vector $\mathbf{O}_{\mathbf{n}}(\varepsilon)$ as

$$
\mathbf{O}_{\mathbf{n}}(\varepsilon)=\left(\begin{array}{c}
O(\varepsilon) \\
\vdots \\
O(\varepsilon)
\end{array}\right)
$$




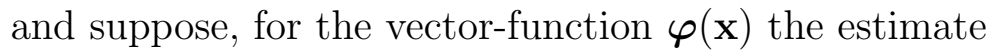

$$
\left|\varphi\left(\mathrm{x}^{\prime}\right)-\varphi\left(\mathrm{x}^{\prime \prime}\right)\right| \leq L\left|\mathrm{x}^{\prime}-\mathrm{x}^{\prime \prime}\right|, \quad \forall \mathrm{x}^{\prime}, \mathrm{x}^{\prime \prime} \in \mathbb{R}^{n}
$$

is satisfied.

From condition (7) and the form of system (5) for solutions with initial data from $\Omega$ we obtain the following relations

$$
\begin{aligned}
& x_{1}(t)=\cos \left(\omega_{0} t\right) x_{1}(0)+O(\varepsilon), \\
& x_{2}(t)=\sin \left(\omega_{0} t\right) x_{1}(0)+O(\varepsilon), \\
& \mathbf{x}_{3}(t)=\exp (\mathbf{A} t) x_{3}(0)+\mathbf{O}_{\mathbf{n}-\mathbf{2}}(\varepsilon)
\end{aligned}
$$

From formulas (8) it follows that for any point $\left(x_{1}(0), x_{2}(0)=0, \mathbf{x}_{3}(0)\right)$, belonging to $\Omega$, there exists a number

$$
T=T\left(x_{1}(0), \mathbf{x}_{3}(0)\right)=2 \pi / \omega_{0}+O(\varepsilon)
$$

such that relations

$$
x_{1}(T)>0, \quad x_{2}(T)=0
$$

are satisfied and conditions

$$
x_{1}(t)>0, x_{2}(t)=0, \quad \forall t \in(0, T)
$$

are not satisfied.

Construct a Poincare map $F$ of the set $\Omega$ for the trajectories of system (5):

$$
F\left\|\begin{array}{c}
x_{1}(0) \\
0 \\
\mathbf{x}_{3}(0)
\end{array}\right\|=\left\|\begin{array}{c}
x_{1}(T) \\
0 \\
\mathbf{x}_{3}(T)
\end{array}\right\| .
$$

Introduce the describing function

$$
\begin{aligned}
& \Phi(a)=\int_{0}^{2 \pi / \omega_{0}}\left[\varphi_{1}\left(\left(\cos \omega_{0} t\right) a,\left(\sin \omega_{0} t\right) a, 0\right) \cos \omega_{0} t+\right. \\
& \left.+\varphi_{2}\left(\left(\cos \omega_{0} t\right) a,\left(\sin \omega_{0} t\right) a, 0\right) \sin \omega_{0} t\right] d t .
\end{aligned}
$$

From estimates (8) and condition on nonlinearity (7) for solutions of system (5) we obtain the following relations

$$
\begin{aligned}
& \left|\mathbf{x}_{3}(T)\right| \leq D \varepsilon \\
& x_{1}^{2}(T)-x_{1}^{2}(0)=2 x_{1}(0) \varepsilon \Phi\left(x_{1}(0)\right)+O\left(\varepsilon^{2}\right)
\end{aligned}
$$

Theorem 1 If the inequalities

$$
\Phi\left(a_{1}\right)>0, \quad \Phi\left(a_{2}\right)<0
$$

are satisfied, then for small enough $\varepsilon>0$ the Poincare map $F$ of the set $\Omega$ into itself is as follows

$F \Omega \subset \Omega$.

From this theorem and the Brouwer fixed point theorem we have the following

Corollary 1 If the inequalities (12) are satisfied, then for small enough $\varepsilon>0$ system (5) has a periodic solution with the period

$$
T=\frac{2 \pi}{\omega_{0}}+O(\varepsilon)
$$

This solution is stable in the sense that its neighborhood $\Omega$ is mapped into itself: $F \Omega \subset \Omega$.

Theorem 2 If the inequalities (12) have opposite signs, then for small enough $\varepsilon>0$ Poincare map (10) of the set $\Omega$ has hyperbolic character: there occurs the contraction with respect to $\mathbf{x}_{3}$ (the estimate (11) is satisfied) and the stretching with respect to $x_{1}: F a_{1}<a_{1}, F a_{2}>a_{2}$. 


\section{Algorithm for determination of stable periodic solutions of gen- erating systems for systems with scalar nonlinearity}

Consider the case of scalar nonlinearity. In this case system (3) takes the form

$$
\frac{d \mathbf{x}}{d t}=\mathbf{P}_{0} \mathbf{x}+\mathbf{q} \varepsilon \varphi\left(\mathbf{r}^{*} \mathbf{x}\right),
$$

where $\mathbf{P}_{0}=\mathbf{P}+k \mathbf{q r}^{*}$ is a constant $n \times n$-matrix, $\mathbf{r}$ and $\mathbf{q}$ are $n$-dimensional vectors, $\varphi(\sigma)$ is a continuous scalar function $(\varphi(0)=0), k$ is a coefficient of harmonic linearization (which is chosen in such a way that matrix $\mathbf{P}_{0}$ has a pair of purely imaginary eigenvalues $\pm i \omega_{0}, \omega_{0}>0$ and the rest of its eigenvalues have negative real parts).

By nonsingular linear transformation, system (13) can be reduced to the form

$$
\begin{aligned}
& \dot{x}_{1}=-\omega_{0} x_{2}+b_{1} \varepsilon \varphi\left(x_{1}+\mathbf{c}^{*} \mathbf{x}_{3}\right), \\
& \dot{x}_{2}=\omega_{0} x_{1}+b_{2} \varepsilon \varphi\left(x_{1}+\mathbf{c}^{*} \mathbf{x}_{3}\right), \\
& \dot{\mathbf{x}}_{3}=\mathbf{A} \mathbf{x}_{3}+\mathbf{b} \varepsilon \varphi\left(x_{1}+\mathbf{c}^{*} \mathbf{x}_{3}\right) .
\end{aligned}
$$

Here $\mathbf{A}$ is a constant $(n-2) \times(n-2)$-matrix, all eigenvalues of which have negative real parts, $\mathbf{b}$ and $\mathbf{c}$ are $(n-2)$-dimensional vectors, $b_{1}$ and $b_{2}$ are certain real numbers.

Now we write the transfer function of system (13):

$$
W_{1}(p)=\mathbf{r}^{*}\left(\mathbf{P}_{0}-p \mathbf{I}\right)^{-1} \mathbf{q}=\frac{\eta p+\theta}{p^{2}+\omega_{0}^{2}}+\frac{R(p)}{Q(p)},
$$

and the transfer function of system (14):

$$
W_{2}(p)=\frac{-b_{1} p+b_{2} \omega_{0}}{p^{2}+\omega_{0}^{2}}+\mathbf{c}^{*}(\mathbf{A}-p \mathbf{I})^{-1} \mathbf{b} .
$$

Here $\eta, \theta$ are certain real numbers, $Q(p)$ is a stable polynomial of degree $(n-2), R(p)$ is a polynomial of degree smaller than $(n-2)$. Suppose, the polynomials $R(p)$ and $Q(p)$ have no common roots. By equivalence of systems (13) and (14) the transfer functions of these systems coincide. This implies the relations

$$
\eta=-b_{1}, \quad \theta=b_{2} \omega_{0}, \quad \frac{R(p)}{Q(p)}=\mathbf{c}^{*}(\mathbf{A}-p \mathbf{I})^{-1} \mathbf{b} .
$$

The following theorems are valid.

Theorem 3 If the conditions

$$
\Phi\left(a_{0}\right)=0,\left.\eta \frac{d \Phi(a)}{d a}\right|_{a=a_{0}}>0
$$

are satisfied, then for small enough $\varepsilon>0$ system (13) with transfer function (15) has T-periodic solution such that

$$
\mathbf{r}^{*} \mathbf{x}(t)=a_{0} \cos \left(\omega_{0} t\right)+O(\varepsilon), \quad T=\frac{2 \pi}{\omega_{0}}+O(\varepsilon) .
$$

This periodic solution is stable in the sense of that there exists its certain $\varepsilon$-neighborhood such that all solutions with the initial data from this $\varepsilon$-neighborhood remain in it with increasing time $t$. 
Theorem 4 If the conditions

$$
\Phi\left(a_{0}\right)=0,\left.\eta \frac{d \Phi(a)}{d a}\right|_{a=a_{0}}<0
$$

are satisfied, then for small enough $\varepsilon>0$ system (13) with transfer function (15) has the solution of the form

$$
\mathbf{r}^{*} \mathbf{x}(t)=a_{0} \cos \left(\omega_{0} t\right)+O(\varepsilon), \quad t \in\left[0, \frac{2 \pi}{\omega_{0}}\right]
$$

and in the neighborhood of this solution the behavior of trajectories has hyperbolic character.

Theorems 3 and 4 coincide with the procedure of search of stable and unstable periodic solutions by means of the harmonic linearization method [|Khalil(2002)|].

Solutions of system (14) have the same form (8) as solutions of system (5). Therefore, for small enough $\varepsilon$ we can obtain the initial data

$$
\mathbf{y}(0)=\left(\begin{array}{c}
y_{1}(0) \\
y_{2}(0) \\
\mathbf{y}_{3}(0)
\end{array}\right)=\left(\begin{array}{c}
a_{0} \\
0 \\
0
\end{array}\right),
$$

where $a_{0}$ is the nonzero root of the equation $\Phi(a)=0$, for the first step of the multistep procedure for localization of hidden oscillation.

\section{Algorithm for attractor localization of Chua's system}

The systems of differential equations, describing the behavior of Chua's circuits [|Chua \& Lin(1990), Chua(1992a), Chua(1992b), Chen \& Ueta(2002), Bilotta \& Pantano(2008)|], are three-dimensional dynamical systems with scalar nonlinearity. Let us apply the above mentioned method to generalized Chua's system [|Leonov et al.(2010)|], represented in dimensionless quantities.

$$
\begin{aligned}
& \dot{x}=\alpha(y-x)-\alpha \psi(x), \\
& \dot{y}=x-y+z, \\
& \dot{z}=-(\beta y+\gamma z) .
\end{aligned}
$$

Here the function

$$
\left.\psi(x)=f(x)+\frac{1}{2}(s-a)\left(\left|x+\delta_{0}\right|-\left|x-\delta_{0}\right|\right)\right)
$$

describes a nonlinear element of system, $\alpha, \beta, \gamma, a$ are parameters of the classical Chua's system, and $\delta_{0}$ and $s$ are parameters that determine the stability of zero equilibrium. The function

$$
\begin{aligned}
& f(x)=m_{1} x+\left(m_{0}-m_{1}\right) \operatorname{sat}(x)= \\
& m_{1} x+\frac{1}{2}\left(m_{0}-m_{1}\right)(|x+1|-|x-1|)
\end{aligned}
$$

represents nonlinear element of classical Chua's system (it is also called Chua's diode). Graphs of nonlinearities (20) and (21) are shown in the Fig. 1. The shaded area is the stability sector. A similar idea of nonlinearity modification was applied in the works [|Suykens et al.(1997), Savaci \& Gunel(2006)|] to increase the number of scrolls of attractor without increase of the system's order.

The procedure described above can be used for numerical localization of attractor of the generalized Chua's system. For this purpose we plug in into system (19) the coefficient $k$ and the small parameter $\varepsilon$ and construct solutions of system (19) with the nonlinearity $\varepsilon \varphi(x)=\varepsilon(\psi(x)-k x)$ by means of sequential increasing $\varepsilon$ with the step 0.1 from the value $\varepsilon_{1}=0.1$ to $\varepsilon_{10}=1$. 


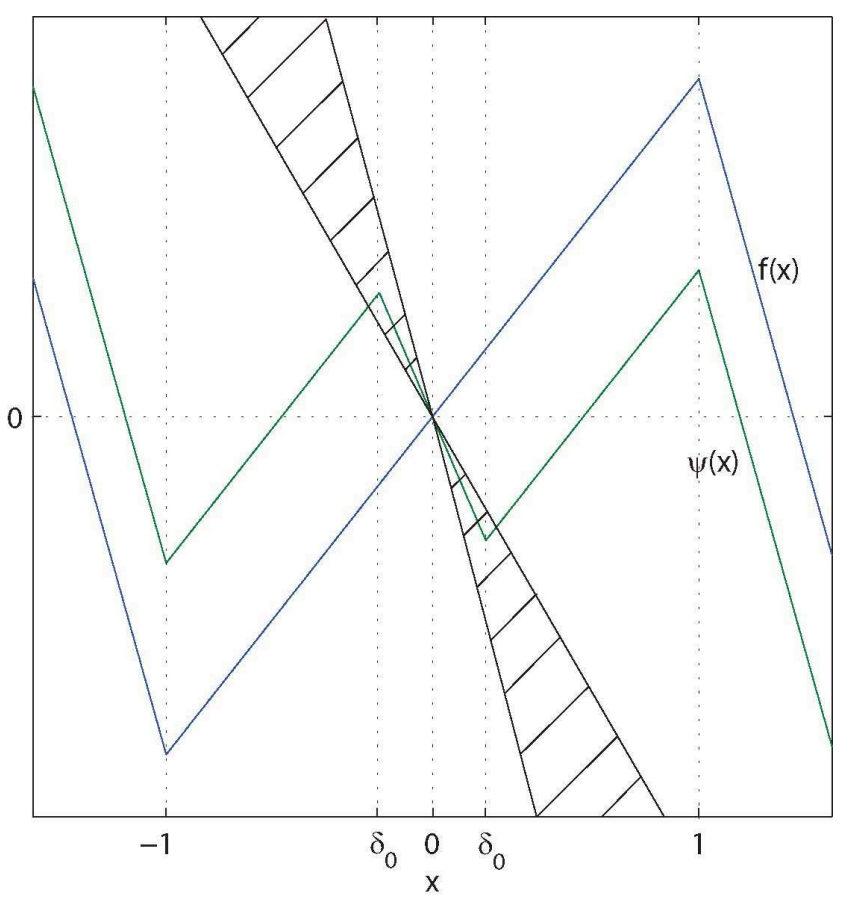

Figure 1: Standard Chua's diode $f(x)$ and modified nonlinearity $\psi(x)$ with stability sector.

Theorem 3 allows to compute the initial data for the system (14). We have to calculate the matrix $\mathbf{S}$ transforming system (13) into (14) to obtain the initial data for the system (13).

Rewrite generalized Chua's system as a Lur'e system

$$
\frac{d \mathbf{x}}{d t}=\mathbf{P} \mathbf{x}+\mathbf{q} \psi\left(\mathbf{r}^{*} \mathbf{x}\right)
$$

where

$$
\mathbf{x} \in \mathbb{R}^{3}, \mathbf{P}=\left(\begin{array}{ccc}
-\alpha & \alpha & 0 \\
1 & -1 & 1 \\
0 & -\beta & -\gamma
\end{array}\right), \mathbf{q}=\left(\begin{array}{c}
-\alpha \\
0 \\
0
\end{array}\right), \mathbf{r}=\left(\begin{array}{l}
1 \\
0 \\
0
\end{array}\right)
$$

Further introduce the coefficient $k$, small parameter $\varepsilon$, and rewrite system (22) in the form of (13)

$$
\frac{d \mathbf{x}}{d t}=\mathbf{P}_{0} \mathbf{x}+\mathbf{q} \varepsilon \varphi\left(\mathbf{r}^{*} \mathbf{x}\right)
$$

where

$$
\begin{aligned}
& \mathbf{P}_{\mathbf{0}}=\mathbf{P}+k \mathbf{q} \mathbf{r}^{*}=\left(\begin{array}{ccc}
-\alpha(1+k) & \alpha & 0 \\
1 & -1 & 1 \\
0 & -\beta & -\gamma
\end{array}\right), \\
& \lambda_{1,2}^{\mathbf{P}_{0}}= \pm i \omega_{0}, \lambda_{3}^{\mathbf{P}_{0}}=-d<0, \varphi(\sigma)=\psi(\sigma)-k \sigma .
\end{aligned}
$$

Using a nonsingular linear transformation $\mathbf{x}=\mathbf{S y}$, it is possible to transform system (23) into (14)

$$
\frac{d \mathbf{y}}{d t}=\mathbf{H y}+\mathbf{b} \varepsilon \varphi\left(\mathbf{u}^{*} \mathbf{y}\right)
$$

where

$$
\mathbf{H}=\left(\begin{array}{ccc}
0 & -\omega_{0} & 0 \\
\omega_{0} & 0 & 0 \\
0 & 0 & -d
\end{array}\right), \mathbf{b}=\left(\begin{array}{c}
b_{1} \\
b_{2} \\
1
\end{array}\right), \mathbf{u}=\left(\begin{array}{c}
1 \\
0 \\
-h
\end{array}\right)
$$


Here the transfer functions $W_{\mathbf{H}}(p)$ of system (24) can be represented as

$$
W_{\mathbf{H}}(p)=\frac{-b_{1} p+b_{2} \omega_{0}}{p^{2}+\omega_{0}^{2}}+\frac{h}{p+d} .
$$

Then, using the equality of transfer functions

$$
W_{\mathbf{H}}(p)=\mathbf{r}^{*}\left(\mathbf{P}_{0}-p \mathbf{I}\right)^{-1} \mathbf{q}
$$

of system (23) and system (24), one can obtain

$$
\begin{aligned}
& k=\frac{-\alpha \gamma+\omega_{0}^{2}-\gamma-\beta}{\alpha(1+\gamma)}, \\
& d=\frac{\alpha+\omega_{0}^{2}-\beta+1+\gamma+\gamma^{2}}{1+\gamma}, \\
& h=\frac{\alpha\left(\gamma+\beta-(1+\gamma) d+d^{2}\right)}{\omega_{0}^{2}+d^{2}}, \\
& b_{1}=\frac{\alpha\left(\gamma+\beta-\omega_{0}^{2}-(1+\gamma) d\right)}{\omega_{0}^{2}+d^{2}}, \\
& b_{2}=\frac{\alpha\left((1+\gamma-d) \omega_{0}^{2}+(\gamma+\beta) d\right)}{\omega_{0}\left(\omega_{0}^{2}+d^{2}\right)} .
\end{aligned}
$$

Since system (23) transforms into system (24) by nonsingular linear conversion $\mathbf{x}=\mathbf{S y}$, therefore, the matrix $\mathbf{S}$ satisfies the following equations

$$
\mathbf{H}=\mathbf{S}^{-1} \mathbf{P}_{\mathbf{0}} \mathbf{S}, \quad \mathbf{b}=\mathbf{S}^{-1} \mathbf{q}, \quad \mathbf{u}^{*}=\mathbf{r}^{*} \mathbf{S} .
$$

Solving these matrix equations, for the matrix

$$
\mathbf{S}=\left(\begin{array}{lll}
s_{11} & s_{12} & s_{13} \\
s_{21} & s_{22} & s_{23} \\
s_{31} & s_{32} & s_{33}
\end{array}\right)
$$

we obtain

$$
\begin{gathered}
s_{11}=1, \quad s_{12}=0, \quad s_{13}=-h, \\
s_{21}=1+k, \quad s_{22}=-\frac{\omega_{0}}{\alpha}, \quad s_{23}=\frac{h(d-k \alpha-\alpha)}{\alpha}, \\
s_{31}=\frac{k \alpha-\omega_{0}^{2}}{\alpha}, \quad s_{32}=-\frac{\left(\alpha \beta+k \alpha \beta+k \alpha \gamma-\gamma \omega_{0}^{2}\right)}{\alpha \omega_{0}}, \\
s_{33}=-\frac{h\left(d^{2}-(1+\alpha+k \alpha) d+k \alpha\right)}{\alpha} .
\end{gathered}
$$

By (18) we obtain the relations between the initial data of systems (23) and (24)

$$
\mathbf{x}(0)=\mathbf{S y}(0)=\mathbf{S}\left(\begin{array}{c}
a_{0} \\
0 \\
0
\end{array}\right)=\left(\begin{array}{c}
a_{0} s_{11} \\
a_{0} s_{21} \\
a_{0} s_{31}
\end{array}\right) .
$$

Thus, using the obtained relations for initial data and applying the multistage algorithm described above, we can numerically simulate Chua's system, represented in the form (13). Returning to the notions of Chua's system, we obtain the following formula for defining initial data:

$$
x(0)=a_{0}, \quad y(0)=a_{0}(1+k), \quad z(0)=a_{0} \frac{k \alpha-\omega_{0}^{2}}{\alpha} .
$$

Consider an example $s=-0.31, \delta_{0}=0.2, a=0.1691, b=-0.4768, \alpha=-1.398, \beta=-0.0136, \gamma=$ -0.0297 . 
Firstly, we compute the coefficient of harmonic linearization $k=-0.3067$ and the value of "start" frequency $\omega_{0}=-0.6436$. Using relations $(25)$ we obtain initial data $x(0)=-1.1061, y(0)=-0.7669, z(0)=0.0115$ for the first step of multistage procedure of construction of solutions. For $\varepsilon_{1}=0.1$ after transient process the computational procedure arrives at a periodic solution close to harmonic one. Further, with increasing parameter $\varepsilon$ this periodic solution close to harmonic one is transformed into chaotic attractor of the type "double-scroll" [|Bilotta \& Pantano(2008)|].

In the classical Chua's system there occurs classical excitation of oscillations in the case when a trajectory from the neighborhood of unstable zero equilibrium reaches the attractor. In this system, in despite of the existence of stable zero equilibrium, the described procedure also allows one to go on "hidden" attractor by means of sequential approximations. The projections of solutions on the plane $\{x, y\}$ for the values $\varepsilon_{1}=0.1$,
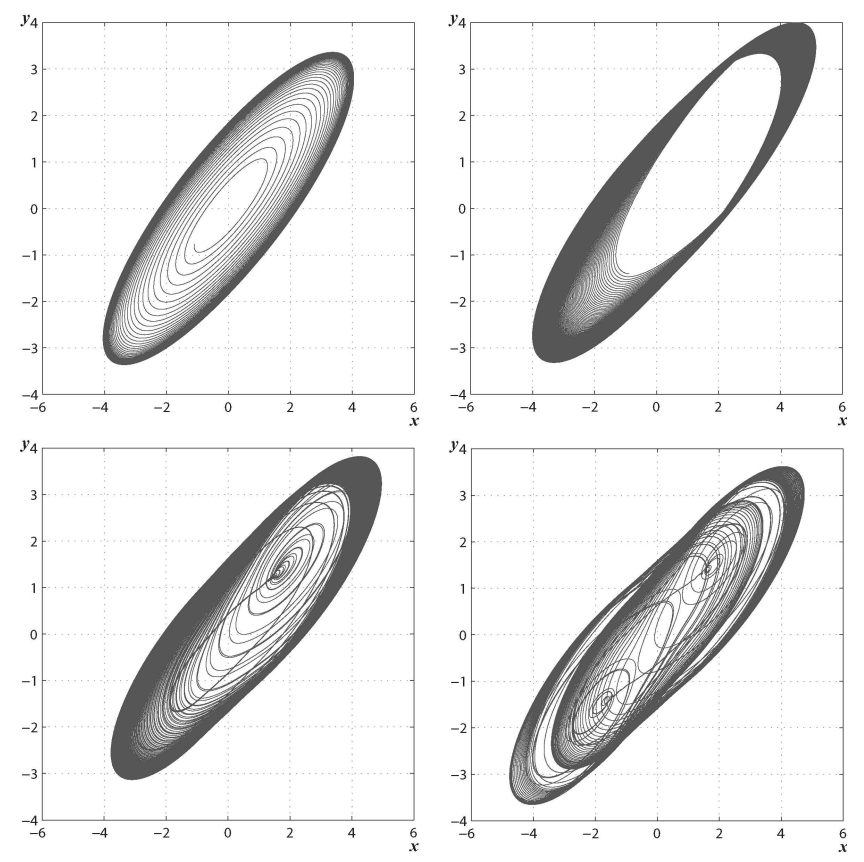

Figure 2: $\varepsilon=0.1, \varepsilon=0.3, \varepsilon=0.7, \varepsilon=1$

$\varepsilon_{3}=0.3, \varepsilon_{7}=0.7$, and $\varepsilon_{10}=1$, respectively are shown in the Fig. 2 .

\section{References}

[Barboza \& Chua(2008)] R. Barboza, L.O. Chua. The Four-Element Chuas Circuit. International Journal of Bifurcation and Chaos, Vol. 18, No. 4, 943-955, 2008.

[Bilotta \& Pantano(2008)] E. Bilotta, P. Pantano. A gallery of Chua attractors World scientific series on nonlinearscience. Series A. Vol. 61, 2008.

[Chen \& Ueta(2002)] G. Chen, T. Ueta. Chaos in Circuits and Systems World scientific series on nonlinear science. Series B. Vol. 11, 2002.

[Chua \& Lin(1990)] L.O. Chua, G.-N. Lin. Canonical Realization of Chua's Circuit Family IEEE Transactions on Circuits and Systems, Vol. 37, No.4, 885-902, 1990.

[Chua(1992a)] L. O. Chua. The Genesis of Chua's Circuit. Archiv fur Elektronik und Ubertragungstechnik, 46, 250-257, 1992. ${ }^{a}$ 
[Chua(1992b)] L.O. Chua. A Zoo of Strange Attractors from the Canonical Chua's Circuits Proceedings of the 35th Midwest Symposium on Circuits and Systems, IEEE, Vol.2, 916-926, 1992. ${ }^{b}$

[Khalil(2002)] H.K. Khalil. Nonlinear Systems. Prentice Hall, New Jersey, 2002.

[Leonov et al.(2010)] G.A. Leonov, V.I. Vagaitsev, N.V. Kuznetsov. Algorithm for localizing Chua attractors based on the harmonic linearization method. Doklady Mathematics, Vol. 82, No. 1, 663-666, 2010.

[Leonov et al.(2010)] G.A. Leonov, V.O. Bragin, N.V. Kuznetsov. Algorithm for Constructing Counterexamples to the Kalman Problem. Doklady Mathematics, Vol. 82, No. 1, 540-542, 2010.

[Matsumoto(1984)] T. Matsumoto. A Chaotic Attractor from Chuas Circuit IEEE Transactions on Circuits and Systems, Vol. CAS-31. No.12, 1055-1058, 1984.

[Savaci \& Gunel(2006)] F.A. Savaci, S. Gunel. Harmonic Balance Analysis of the Generalized Chua's Circuit. International Journal of Bifurcation and Chaos, Vol. 16, No. 8, 2325-2332, 2006.

[Suykens et al.(1997)] J.A.K. Suykens, A. Huang, L.O. Chua. A Family of n-scroll Attractors from a Generalized Chuas Circuit AEU-International Journal of Electronics 83 Communications, Vol. 51, No. 3, 131-138, 1997. 